\title{
A role for the alveolar epithelium in recruitment of mononuclear cells into the lung
}

\author{
David M. Center
}

Pulmonary Center and Pulmonary and Critical Care Division, R-304, Boston University Medical Center, Boston University School of Medicine, 715 Albany Street, Boston, Massachusetts 02118, USA. Phone: (617) 638-4860; Fax: (617) 536-8093; E-mail: dcenter@lung.bumc.bu.edu.

Just as viral infection of the respiratory epithelium results in injury and death to the host cells, the defensive measures available to the host also damage the lung, causing injury that is not restricted to the infected cells. These host responses occur in several stages, beginning when viral infection induces the infected host cell to synthesize and to secrete type 1 IFNs and chemokines, such as IL-8 (1). The resulting influx of neutrophils, cells that are capable of neither target cell specificity nor memory, causes proteolytic and oxidative tissue damage affecting virus-infected cells and their uninfected neighbors alike.

Only at a later stage does the precision of the host response improve, as viral infection stimulates both $\mathrm{CD}^{+}$cytotoxic $\mathrm{T}$-cell responses and antibody production. The antibody response helps limit the infection by facilitating the early and specific destruction of virus-infected cells, through antibody-dependent cytotoxicity mediated by mononuclear phagocytes. Further selectivity toward infected cells comes from the adaptive immune response by $\mathrm{CD}^{+}$cytotoxic $\mathrm{T}$ cells, which recognize viral antigens expressed in concert with MHC I target host cell antigens on the epithelial cell surface. Target cell lysis occurs only if viral antigens are expressed in concert with MHC I similar to that of the effector cells (2).

Thus, viral infection in an immune host initiates a race in which cytotoxic $T$ cells compete to destroy cells supporting viral replication before the virus can spread to enough cells to irreparably damage the host (3). Unfortunately for the host, it matters little whether the destruction of vital epithelium is caused by virus, oxidative stress of products of the innate immune system, or $\mathrm{CD}^{+}$ cytotoxic cells.

\section{Antiviral defenses without viruses}

In this issue of the JCI, Richard Enelow's laboratory identifies yet another phase of the cytotoxic response to viral infection of the airway epithelium. During this late phase, antigenspecific, MHC I-restricted $\mathrm{CD}^{+} \mathrm{T}$ cells activate the target epithelium to synthesize and to secrete chemokines that attract macrophages to the tissue (4). Using an elegant transgenic mouse model (5), Zhao et al. (4) have been able to observe epithelial damage caused by immune cytotoxicity free of any direct epithelial damage occurs 72-96 hours after transfer and is associated with a massive influx of host macrophages.

The system is somewhat artificial in that it is unlikely that in any viral infection all type II pneumocytes would be infected targets for cytotoxicity. Nevertheless, the lessons are profound and provocative. The authors demonstrated that the macrophage influx is antigenand MHC I-restricted and that cytotoxic damage depends upon TNF- $\alpha$ signaling by effector cells. Recipient mice lacking the receptor TNF- $\alpha \mathrm{R} 1$, accordingly, experience no alveolar damage. The source of the macrophage chemotactic signals is the alveolar epithelium expressing viral antigen transgenes. The expression of chemokines by respiratory epithelium is not novel (6), but the mode of induction is unique. Of note, the TNF- $\alpha$ signal does not invariably lead to death, but can also be accompanied by an NF-KB-dependent activation signal that induces expression of the chemokines macrophage inflammatory protein-2 (MIP-2) and monocyte chemoattractant protein-1 (MCP-1). It remains uncertain cell killing caused by the virus itself. By placing an influenza virus antigen under the control of the promoter for a lung surfactant protein gene, they have created mice in which this antigen, but no other viral components, is constitutively expressed in type II pneumocytes and the lower respiratory epithelium.

Adoptive transfer into these transgenic mice of syngeneic memory $\mathrm{CD}^{+}$ T cytotoxic cells specific for the viral epitope causes cell dose-dependent damage to the epithelium. The kinetics of this response are of considerable interest. The exogenous $\mathrm{CD}^{+}$cells can be found in the lung within 3 hours of transfer and are cleared from the lung within 48 hours. However, the majority of the whether these two TNF- $\alpha$ responses induction of caspases and cell death, or induction of NF- $\kappa B$ and chemokines - occur in distinct populations of target cells or in the same cells at different stages of the process. Differential activation of the relevant signaling events could provide biochemical insight into the cellular specificity of these responses (7). In either case, however, the current work has defined a third, macrophagedependent mechanism of host defense against viruses that leads to an unusual form of communal cellular suicide, a mechanism that is initiated by virusinfected cells only after they have been targeted for death by cytotoxic T cells. 
In addition, Zhao et al. (4) find that the chemokine MCP-1 mRNA is induced in the transgenic epithelium in under 3 hours following adoptive transfer of cytotoxic T cells. Thus, in an otherwise normal lung, cytotoxic $\mathrm{T}$ cells are clearly able to migrate through endothelium and interstitium and reach the alveolar epithelial cells that express the viral antigen. Moreover, these $\mathrm{T}$ cells must reach their target in sufficient numbers and provide enough stimulation to the TNF- $\alpha$ signaling pathway that the latter cells are efficiently induced to transcribe MCP-1. Because all of these events must occur within 3 hours, there appears to be little time in which to initiate the migration of cytotoxic $\mathrm{T}$ cells toward the target epithelium, suggesting that influx and efflux of at least some $\mathrm{T}$ cells through the lung parenchyma occur constitutively in these animals, even in the absence of local inflammation.

\section{Explaining the kinetics of the $\mathrm{T}$-cell response}

Three possible explanations for this rapid response come to mind. First, it may be incorrect to assume that no local inflammation exists. There may always be some inflammation in the lung, given the enormous antigen load borne by the thousands of liters of gas exchanged each day. Subclinical inflammation provides the signals necessary for conversion of normal pulmonary endothelium into one that is susceptible to adherence and transmigration of T cells. This model seems unlikely because the lung would soon become inundated with leukocytes, which would be detrimental to gas exchange.

As a second model that might account for the remarkable efficiency of T-cell interactions with the alveolar epithelium, influx and efflux of T cells through the lung parenchyma might occur as part of a normal homeostatic mechanism in this tissue. Random migration of these cells might provide the necessary critical mass of contact with $\mathrm{T}$ cells to initiate the epithelial cell responses. If so, this robust recirculation through lung parenchyma might be explained if lower respiratory $\mathrm{T}$ cells have a motile phenotype (8) and predilection for lung, perhaps mediated by lung-specific chemokines or $\mathrm{CD}^{+}$cell-borne chemokine recep- tors. Chemokines could provide all the signals necessary to induce T-cell attachment to, and migration across, the lung endothelium and lung-associated lymphoid tissues and could create a chemotactic gradient drawing them to the epithelial cells (9-11).

Consistent with this suggestion, $\mathrm{T}$ cells are found in the broncho-alveolar compartment in all normal mammals, albeit in very small numbers. However, it is not clear whether these cells are present because of low levels of persistent inflammation or because of homing through normal lung, nor does their presence give insights into the mechanism of lung transmigration. In light of our most recent understanding of the essential role of chemokine/chemokine receptors in defining the lymphoid organ anatomy $(11,12)$ and in homing of memory $\mathrm{T}$ cells to skin and gut (13-15), it is likely that a similar system determines the migration of lymphocytes through lung. The kinetics of the responses observed in the paper by Zhao et al. (4) predict that such a pattern will emerge. If so, the factors that permit efflux of lung-specific lymphocytes must be equally important for lymphocyte recirculation but remain completely obscure. However, we must exercise caution in extrapolating recirculation patterns derived from work in rodents, which have an abundant bronchus-associated lymphoid network, to humans, a species with far less prominent local lung lymph node architecture.

A third possible mechanism by which $T$ cells might home to sites of antigen presentation in an efficient and specific manner would require that viral antigens expressed on the cell surface, either alone or associated with MHC molecules, are shed from the alveolar epithelium and create a chemotactic gradient centered on the target tissue. Indirect support for this proposal lies in the observations that antibodies to CD3 are chemotactic for $\mathrm{T}$ cells (16) and that antigen itself can be chemotactic (17). Considering the vascular cross-sectional area of organs like lung and skin, it seems more likely that sufficient numbers of antigenspecific cells would be attracted to an area in which antigen has been introduced by the presence of a specific chemotactic gradient, rather than by chemokine stimulation of bulk migra- tion by all lung-specific $\mathrm{T}$ cells. If processed antigen, with or without MHC, were distributed in such a chemotactic gradient, it would not be hard to imagine recruitment to any remote site in an expeditious manner. Clearly, if both antigen and chemokine gradients coexisted, cell localization could be still more efficient.

\section{Is the cure worse than the disease?}

Regardless of the mechanism of T-cell localization in this model, it seems clear that the host first initiates a nonspecific antiviral response, and then a target-cell specific cytotoxic response, which prompts yet a third, macrophage-mediated nonspecific response. Why this seemingly unnecessary and destructive final step is beneficial remains puzzling. However, it can be speculated that in natural influenza infection, the host might attempt to limit virus to the portal of entry in the upper respiratory tract by deploying IFNs and chemokineinduced neutrophils to the infected area. Targets of cytotoxic $\mathrm{T}$ cells would provide a second wave of removal of virus-infected cells if virus persists. As virus spreads beyond the upper airways, either by local extension or following viremia, the cytotoxic T-cell response and target-cell dependent, chemokine-mediated macrophage response would help prevent universal involvement of lower respiratory tract and gas exchange units. In this case, the tertiary response identified by Zhao et al. (4) might be the last stand in preventing further advancement of virus. In this regard, one wonders if lung epitheli$\mathrm{um}$ is unique in the late chemokinedependent macrophage response and whether natural virus infection alters the complexion and complexity of the chemotactic milieu. Further, we need to confirm the presence of this response in natural human infection. It will be important to determine whether the alveolar epithelium is the source of chemoattractant factors that regulate alveolar macrophages and intraepithelial lymphocyte traffic and homing (15) during normal homeostasis, as may be the case in the gut (13) and skin (14). If the cure is sometimes worse than the disease, perhaps the current work has identified important new targets for therapeutics during acute lung injury fol- 
lowing viral infection: macrophagedependent damage of the epithelium and the chemokine-signaling pathways that induce this response.

1. Fiedler, M.A., Wernke-Dollries, K., and Stark, J.M. 1995. Respiratory syncytial virus increases IL-8 gene expression and protein release in A549 cells. Am. J. Physiol. 269:L865-L872.

2. Zinkernagel, R.M., and Doherty, P.C. 1974. Restriction of in vitro T cell-mediated cytotoxicity in lymphocytic choriomeningitis within a syngeneic or semiallogeneic system. Nature. 248:701-702.

3. Doherty, P.C. 1998. The numbers game for virusspecific CD8+ T cells. Science. 280:227.

4. Zhao, M.Q., et al. 2000. Alveolar epithelial cell chemokine expression triggered by antigen-specific cytolytic CD8 ${ }^{+} \mathrm{T}$ cell recognition. J. Clin. Invest. 106:R49-R58.

5. Liu, A.N., et al. 1999. Perforin-independent cytotoxicity in T cell-mediated cytotoxicity of alveolar epithelial cells is preferentially mediated by tumor necrosis factor- $\alpha$ : relative insensitivity to Fas ligand. Am. J. Respir. Cell Mol. Biol. 20:849-858.

6. Standiford, T.J., et al. 1990. Interleukin-8 gene expression by a pulmonary epithelial cell line: a model for cytokine networks in the lung. J. Clin. Invest. 86:1945-1953.

7. Pomerantz, J.L., and Baltimore, D. 2000. A cellular rescue team. Nature. 406:26-29.

8. Masuyama, J., Berman, J.S., Cruikshank, W.W. Morimoto, C., and Center, D.M. 1992. Evidence for recent as well as long term activation of $\mathrm{T}$ cells migrating through endothelial cell monolayers in vitro. J. Immunol. 148:1367-1374.

9. Pachynski, R.K., Wu, S.W., Gunn, M.D., and Erle, D.J. 1998. Secondary lymphoid-tissue chemokine (SLC) stimulates integrin alpha 4 beta 7-mediated adhesion of lymphocytes to mucosal addressin cell adhesion molecule-1 (MAdCAM-1) unde flow. J Immunol. 161:952-956.

10. Stein, J.V., et al. 2000. The CC chemokine thymus-derived chemotactic agent 4 (TCA-4, secondary lymphoid tissue chemokine, $6 \mathrm{Ckine}$, exodus-2) triggers lymphocyte function-associated antigen 1-mediated arrest of rolling $\mathrm{T}$ lymphocytes in peripheral lymph node high endothelia venules. J. Exp. Med. 191:61-76.

11. Ngo, V.N., et al. 1999. Lymphotoxin alpha/beta and tumor necrosis factor are required for stromal cell expression of homing chemokines in $\mathrm{B}$ and $\mathrm{T}$ cell areas of the spleen. J. Exp. Med. 189:403-412 12. Cyster, J.G., et al. 1999. Chemokines and B-cell homing to follicles. Curr. Top. Microbiol. Immunol. 246:87-92.

13. Zabel, B.A., et al. 1999. Human G protein coupled receptor GPR-9-6CC chemokine receptor 9 is selectively expressed on intestinal homing $\mathrm{T}$ lymphocytes, mucosal lymphocytes and thymocytes and is required for thymus expressed chemokine mediated chemotaxis. J. Exp. Med. 190:1241-1251.

14. Morales, J., et al. 1999. CTACK, a skin associated chemokine that preferentially attracts skin homing memory T cells. Proc. Natl. Acad. Sci. USA. 96:14470-14474.

15. Erle, D.J., and Pabst, R. 2000. Intraepithelial lymphocytes in the lung: a neglected lymphocyte population. Am. J. Respir. Cell Mol. Biol. 22:398-400.

16. Ryan, T.C. Cruikshank, W.W. Kornfeld, H Collins, T.L., and Center, D.M. 1995. The CD4 associated tyrosine kinase p56lck is required for lymphocyte chemoattractant factor-induced $T$ lymphocyte migration. J. Biol. Chem. 270:17081-17086.

17. Wilkenson, P.C., Parrott, D.M.V., Russell, R.J., and Sless, F. 1977. Antigen-induced locomotor responses in lymphocytes. J. Exp. Med. 145:1158-1168. 